\title{
Adult outcomes of conduct problems in childhood or adolescence: further evidence of the societal burden of conduct problems
}

\author{
Graeme Fairchild ${ }^{1}$
}

Published online: 4 September 2018

c) Springer-Verlag GmbH Germany, part of Springer Nature 2018

Conduct problems are common and costly to society, with recent studies documenting dramatically increased levels of adult service use in those with conduct problems in childhood [1]. Conduct problems have been found to predict a range of mental and physical health problems, and poorer educational, occupational, and interpersonal outcomes. They also place a substantial burden on the affected individuals, their families, and their local communities [2, 3]. There is broad agreement in the literature that conduct problems are highly heterogeneous in terms of their symptoms, aetiology, functional impact, and developmental course, and therefore, some way of parsing this heterogeneity by identifying meaningful subtypes is required. One prominent subtyping approach has been to classify conduct problems according to their age-of-onset-for example, the DSM-5 distinguishes between childhood-onset and adolescence-onset Conduct Disorder according to the presence or the absence of conduct problems prior to age 10 [4]. The availability of repeated-measures data from prospective longitudinal studies investigating the development of antisocial behaviour and advanced statistical methods for modelling group-based trajectories has led to a dramatic expansion in our knowledge regarding the developmental course of conduct problems. These methods have also enabled researchers to study the causes, correlates, and outcomes of different conduct problem trajectories.

The paper by Bevilacqua and colleagues in the present edition of ECAP provides a highly readable and informative introduction to this literature on the developmental trajectories of antisocial behaviour and theories seeking to explain them such as the developmental taxonomic theory [5] (see also [6] for a recent overview). The authors performed a systematic review and quantitative meta-analysis to examine the

Graeme Fairchild

g.fairchild@bath.ac.uk

1 Department of Psychology, University of Bath, Bath, Somerset BA2 7AY, UK early adult outcomes of each of the three elevated conduct problems trajectories, to investigate whether the life-course persistent (also known as the 'early-onset persistent') trajectory is associated with the worst functional outcomesconsistent with the developmental taxonomic theory. The authors also tested whether the childhood-limited or adolescence-onset trajectories (which are frequently found to be the most common elevated trajectories in population-based samples) are associated with significantly poorer outcomes than the low conduct problems trajectory. Their systematic review highlighted that there are surprisingly few published longitudinal studies investigating adult outcomes of conduct problems trajectories $(n=13)$, and only nine studies were eligible for inclusion in their quantitative meta-analysis. It should also be noted that much of the empirical evidence we have in this area comes from a single, albeit highly informative and influential, prospective longitudinal study (the Dunedin Multidisciplinary Health and Development Study), and all published outcome studies in this area were performed in high-income countries such as the US, UK, and New Zealand. The results of the meta-analysis showed that the early-onset persistent trajectory is indeed associated with the poorest health, educational, and social outcomes, but contrary to the developmental taxonomic theory which holds that antisocial behaviour that emerges in adolescence is usually 'adolescence-limited' and relatively benign in its impact, the adolescence-onset trajectory was also linked to significantly poorer outcomes than the low trajectory across multiple domains (e.g., mental and physical health in adulthood, substance abuse, aggression, criminal behaviour, and educational attainment). Strikingly, when individuals on the early-onset persistent and adolescence-onset trajectories were directly compared, there were no significant differences for any of the adult outcomes studied. Interestingly, even the group that desisted from showing conduct problems as they entered late childhood or adolescence - the "childhood-limited' group — had poorer educational outcomes and showed 
significantly higher levels of aggression in adulthood than individuals on the low conduct problems trajectory.

These important findings document the long-term negative impact of conduct problems, regardless of whether they emerge in childhood or adolescence, and highlight the need to provide effective interventions for children with conduct problems and prevention programmes for those at high risk of developing conduct problems. The results also have implications for the future development of the diagnostic and classification system, because they cast doubt on the usefulness of the distinction made in the DSM- 5 between childhood-onset and adolescence-onset forms of Conduct Disorder [4]. The findings also present a challenge to the developmental taxonomic theory, because they provide further evidence that conduct problems which emerge in adolescence often become persistent (rather than being 'limited' to adolescence) and frequently lead to severe impairment. They also complement research demonstrating that both childhood-onset and adolescence-onset forms of Conduct Disorder are associated with neurocognitive impairments and abnormalities in brain structure and function (e.g., lower amygdala volume; see [7] for a review). Nevertheless, further research is needed to investigate whether different subtypes of conduct problems require different interventions (see [8] for suggestions in this respect), and whether individuals with adolescence-onset conduct problems are more responsive to treatment than their early-onset counterparts. Additional research is urgently needed to understand why so many young people grow out of conduct problems and the processes and mechanisms underlying this pattern of desistence. The gap in our knowledge regarding the adult outcomes of different conduct problems trajectories in lowand medium-income countries should also be addressed in future work. Finally, it will be important to maintain the existing longitudinal studies to allow researchers to investigate the outcomes of different conduct problems trajectories into later life, as only the Dunedin Study has continued to assess participants beyond early adulthood (age 45 data collection is now underway).

Another set of papers in this edition of the journal are concerned with efforts to characterize, diagnose, and treat attention-deficit/hyperactivity disorder (ADHD) in children and adults. The papers by Bolte et al. and Mahdi et al. describe efforts to use the World Health Organisation's International Classification of Functioning, Disability and Health (ICF) framework to characterize the impact of ADHD on a broad range of functional domains, and assess strengths associated with ADHD as well as impairments. The aim of the ICF initiative is to document the impact of a given disorder on the patient's functioning in a comprehensive and individualised manner that includes both strengths and disorder-related difficulties. It is hoped that this will inform treatments for ADHD, leading to a more personalised approach, and help guide resource allocation. The first paper from the consortium reports on consensus meetings of international experts seeking to identify the core sets of functioning that should be assessed in individuals with ADHD, and which functional domains should be assessed at each stage of the lifespan. The second paper by Mahdi et al. describes a cross-sectional study which applied the ICF to assess the functioning of children, adolescents, and adults with ADHD in clinical practice settings. The study found that difficulties in initiating and completing multiple tasks, managing time and scheduling activities, and making decisions were amongst the most common areas of weakness in patients with ADHD, beyond those directly related to the diagnostic criteria for ADHD (i.e., attention problems). Strengths associated with ADHD relate to positive personality traits (agreeableness, optimism, and openness to experience), enthusiasm for social activities and creativity (for further qualitative evidence on this issue see [9]). While it is encouraging to see researchers taking a broader perspective on ADHD and considering strengths associated with the condition in addition to weaknesses, the clinical utility of assessing functioning in individuals with ADHD in the detailed manner outlined in the ICF- even the substantially reduced ICF 'Core Sets' - needs to be confirmed before the system is adopted more widely, given the associated time costs and resource implications. It would also be interesting to use the ICF Core Sets framework to assess treatmentrelated improvements in functioning (beyond reduction of core ADHD symptoms). In a further paper related to the treatment of ADHD, Huss and colleagues report data from a phase 3 safety study of extended release guanfacine in children and adolescents with ADHD. The study included children and adolescents from 11 different European countries and documented the incidence of treatment-emergent adverse events across a range of psychological, sleep-related, and physiological domains. The most common adverse events associated with guanfacine treatment were somnolence, headache, and fatigue, and sleep-related side effects peaked in week 3 and declined thereafter. Extended release guanfacine was found to be effective in the treatment of ADHD symptoms, and was well-tolerated by the majority of participants over the study period of 2 years. This study provides important information about the typical profile and time course of side effects related to extended release guanfacine treatment, as well as the efficacy of such treatment in reducing ADHD symptoms, which may be helpful for clinicians considering prescribing guanfacine to their patients in the future as an alternative to stimulant-based medications.

In summary, several papers published in the present edition of ECAP make important contributions to our understanding of conduct problems and ADHD. Although conduct problems and, to a lesser extent, ADHD are under-recognized and understudied, it is encouraging to see researchers 
making progress on several fronts in terms of documenting the long-term impact of these conditions, understanding the wider context in which they emerge and are maintained, and developing and testing new interventions.

\section{References}

1. Rivenbark JG, Odgers CL, Caspi A, Harrington H, Hogan S, Houts RM, Poulton R, Moffitt TE (2018) The high societal costs of childhood conduct problems: evidence from administrative records up to age 38 in a longitudinal birth cohort. J Child Psychol Psychiatry 59(6):703-710

2. Roberts R, McCrory E, Joffe H, De Lima N, Viding E (2018) Living with conduct problem youth: family functioning and parental perceptions of their child. Eur Child Adolesc Psychiatry 27(5):595-604

3. Erskine HE, Ferrari AJ, Polanczyk GV, Moffitt TE, Murray CJ, Vos T, Whiteford HA, Scott JG (2014) The global burden of conduct disorder and attention-deficit/hyperactivity disorder in 2010. J Child Psychol Psychiatry 55(4):328-336

4. American Psychiatric Association (2013) Diagnostic and statistical manual of mental disorders, 5th edition (DSM-5). American Psychiatric Publishing, Arlington

5. Moffitt TE (1993) Adolescence-limited and life-course-persistent antisocial behavior: a developmental taxonomy. Psychol Rev 100(4):674-701

6. Moffitt TE (2018) Male antisocial behaviour in adolescence and beyond. Nat Hum Behav 2:177-186

7. Fairchild G, van Goozen SH, Calder AJ, Goodyer IM (2013) Research review: evaluating and reformulating the developmental taxonomic theory of antisocial behaviour. J Child Psychol Psychiatry 54(9):924-940

8. Klahr A, Burt SA (2014) Practitioner Review: Evaluating the known behavioral heterogeneity in conduct disorder to improve its assessment and treatment. J Child Psychol Psychiatry 55(12):1300-1310

9. Mahdi S, Viljoen M, Massuti R, Selb M, Almodayfer O, Karande S, de Vries PJ, Rohde L, Bölte S (2017) An international qualitative study of ability and disability in ADHD using the WHO-ICF framework. Eur Child Adolesc Psychiatry 26(10):1219-1231 